\title{
Stellar evolution constrains primordial black holes as dark matter candidates
}

\section{F. Capela}

DAMPT, Centre for Mathematical Sciences, Cambridge University, Wilberforce Road, Cambridge CB3 OWA, U.K.

\section{M.S. Pshirkov*}

Sternberg Astronomical Institute, Lomonosov Moscow State University, Universitetsky prospekt 13, 119992, Moscow, Russia

Institute for Nuclear Research of the Russian Academy of Sciences, 117312, Moscow, Russia Pushchino Radio Astronomy Observatory, 142290 Pushchino, Russia

E-mail: Dshirkovdsai.msu.ru

\section{P. G. Tinyakov}

Université Libre de Bruxelles, Service de Physique Théorique, CP225, 1050, Brussels, Belgium

\begin{abstract}
By considering adiabatic contraction of the dark matter (DM) during star formation, we estimate the amount of DM trapped in stars at their birth. If the DM consists partly of primordial black holes (PBHs), they will be trapped together with the rest of the DM and will be finally inherited by a star compact remnant - a white dwarf (WD) or a neutron star (NS), which they will destroy in a short time. Observations of WDs and NSs thus impose constraints on the abundance of PBH. We show that the best constraints come from WDs and NSs in globular clusters which exclude the DM consisting entirely of PBH in the mass range $10^{16}-10^{22} \mathrm{~g}$, with the strongest constraint on the fraction $\Omega_{\mathrm{PBH}} / \Omega_{\mathrm{DM}} \leq 10^{-4}$ being around PBH masses $10^{21} \mathrm{~g}$.
\end{abstract}

The 34th International Cosmic Ray Conference,

30 July- 6 August, 2015

The Hague, The Netherlands

\footnotetext{
*Speaker.
} 


\section{Introduction}

There are compelling evidences that almost $30 \%$ of the energy density of the Universe is contained in the form of a non-relativistic non-baryonic dark matter (DM) [W]. The DM nature remains essentially unconstrained, leaving room for a host of candidates. It is frequently assumed that the DM is composed of some kind of new particles beyond the Standard Model of particle physics. However, other candidates such as primordial black holes ( $\mathrm{PBH}$ ) may provide a viable alternative: the obvious advantage is that no new particles beyond the extemely well tested Standard Model are required.

Stars, especially their remnants, could be used as a very sensitive probe to this kind of dark matter candidates: in higher density environment of compact stars stars, the accretion on even smlall black holes is sufficiently efficient to destroy the star in a short time (see Ref. [D]. Even if a single PBH is captured by a compact star, the latter gets destroyed. Requiring that the probability of such an event is small leads to the constraints on the PBH abundance [B], 团].

The strength of the constraints is determined by the amount of captured DM (firstly we well speak about DM in general, discussing the particular PBH case later). There are two different capture mechanisms. A star can capture DM from its surrounding environment, such as the Galactic halo, during its lifetime, including a compact remnant stage. The DM particles passing through the star may interact with the nucleons, losing enough energy to become gravitationally bound [ [5], 目]. Then each subsequent orbit will also pass through the star, so that eventually, after many collisions, the DM particle will sink to the center of the star. Such capture process can lead to the accumulation of a considerable amount of DM inside the compact star throughout its lifetime [ $[\mathbf{D}]$.

Also, the DM could also be captured during the star formation. In the course of gravitational collapse of a prestellar core in a giant molecular cloud, the DM that was initially gravitationally bound to the core undergoes adiabatic contraction, forming a cuspy profile centered at the star, with the density $\rho(r)$ behaving like $\rho(r) \propto r^{-3 / 2}$. We are interested, however in a amount of DM captured by a star as a result of its formation. The usually considered DM density profile after the contraction is not sufficient for that purpose, and one needs to know in more detail the distribution of DM in the phase space.

To get captured, the DM particles have to lose their energy by interactions with the star material. Therefore, one needs to calculate the number of particles whose orbits cross the star after the adiabatic contraction and, what is crucial, to account correctly for particles that spend only a small fraction of time inside the star, because they consitute the ovewhelming majority of all particles.

\section{Adiabatic contraction of DM during star formation}

\subsection{Star formation and adiabatic contraction of DM}

Stars are formed in giant molecular clouds (GMCs) with a typical mass of $10^{5}-10^{6} M_{\odot}$ and density $\rho_{B} \sim 500 \mathrm{GeV} / \mathrm{cm}^{3}$. Eventually, GMCs fragment into smaller clumps, each forming a protostar after the contraction of the gas. In these regions baryonic densities are quite high, so for most environments the gravitational effect of the DM on the formation of stars is negligible and the behavior of DM is determined by the gravitational potential of the baryons. 
The baryons contracting into a protostar create a deepening gravitational potential which drags the DM particles along - the DM distribution develops a density profile that is peaked at the core of the prestellar cloud.

\subsection{Simulation of DM orbits}

The core parameters were taken from the Ref. [团], the typical values being the baryonic density $\rho \sim 5 \times 10^{6} \mathrm{GeV} / \mathrm{cm}^{3}$ and the size $\sim 5000 \mathrm{AU}$.

In the adiabatic approximation only the initial and final states matter. The gravitational potential of the contracting baryons was modeled as a two-component mass distribution: the uniform spherical cloud and a point mass in its center. The total mass of the cloud and the value of the central mass are chosen in such a way that the latter linearly grows from zero at $t=0$ to the maximum value at $t=T$, while the former decreases from the maximum value to zero, the total mass of the system being constant. The change of mass was performed slowly over a period of time $T$ that is at least several times longer than free fall time $t_{\mathrm{ff}}$ in order for adiabatic condiions to be satisfied.

DM was initially distributed uniformly in space (characterized by some background DM density $\bar{\rho}_{\mathrm{DM}}$ ), and had Maxwellian distribution in velocities with the dispersion $\bar{v}$,

$$
d n=\bar{n}_{\mathrm{DM}}\left(\frac{3}{2 \pi \bar{v}^{2}}\right)^{3 / 2} \exp \left\{\frac{-3 v^{2}}{2 \bar{v}^{2}}\right\} d^{3} v,
$$

where $\bar{n}_{\mathrm{DM}}=\rho_{\mathrm{DM}} / m_{\mathrm{DM}}$ is the mean DM number density. An estimate of the initial density of DM that is gravitationally bound inside the pre-stellar core has been computed for several star masses in [䧃]. From eq. ([2.]), at zero velocity $v$ the ambient phase space density is (cf. [8])

$$
\mathscr{Q}_{\max }=\left(\frac{3}{2 \pi}\right)^{3 / 2} \frac{\bar{\rho}_{\mathrm{DM}}}{m_{\mathrm{DM}}^{4} \bar{v}^{3}} .
$$

The density of DM gravitationally bound to the pre-stellar core is smaller as compared to the ambient DM density by the factor $(v / \bar{v})^{3} \ll 1$, $v$ being the escape velocity from the pre-stellar core.

The simulation proceeds as follows: at $t=0$ we inject a DM particle with a random uniformly distributed initial position and velocity. If the particle is gravitationally bound at $t=0$, it is evolved through the equations of motion, otherwise it is rejected; $3 \times 10^{7}$ trajectories were simulated in this way.

The density profile can be obtained by recording the positions of particles at a randomly chosen time $t>T$ after the end of the contraction. The distribution in $r$ can be then converted into particle density. An accurate estimate of the number of captured particles, however, needs additional information, because many trajectories pass through the star, but spend most of their time outside. We calculate and record the periastron $r_{\min }$ and apastron $r_{\max }$ of each particle orbit at the end of star formation to get full information about particle trajectory. The distribution of $r_{\text {min }}$ determines the number of particles that ever get within a given distance from the center and could be eventually captured.

Fig. W shows the resulting distributions as a function of $r / \bar{R}$, where $\bar{R}$ is the initial radius of the cloud. The actual star radius is also indicated on the plot. The lower curve shows the fraction $n(r)$ of particles that are, in a given moment of time, within the radius $r$ from the center. Points with errors 
Figure 1: Lower curve: The fraction of particles $n(r)$ that are found within radius $r$ at the end of the adiabatic contraction. $\bar{R}$ is the initial radius of the prestellar core. Upper curve: The fraction of particles $v(r)$ whose orbits have the periastron smaller than $r$. Lines show the power laws $n(r) \propto r^{1.5}$ and $v(r) \propto r$. The errorbars represent statistical errors (adopted from [Q]]).

represent the results of the simulation. The straight line is a fit by the power law $n(r) \propto r^{1.5}$. As $n(r)$ is proportional to the density integrated up to the radius $r$, we can conclude that $\rho(r) \propto r^{-1.5}$, in agreement with the results of Refs. [四] and [ए]]

The upper curve shows the fraction $v(r)$ of particles whose orbits have periastra within the radius $r$. The fraction of such orbits is larger than $n(r)$ : after the adiabatic contraction, a considerable amount of DM particles have very elongated orbits and spend most of their time at radii larger than $r$. Indeeed, $v(r)$ scales differently, $v(r) \propto r$. The ratio of the two curves gives the enhancement factor as a function of the radius. At the star radius $r=R_{\odot}$ this factor is $v\left(R_{\odot}\right) / n\left(R_{\odot}\right)=1.84 \times 10^{3}$.

To summarize, as a result of the formation of a star, the DM bound to the prestellar core experiences the adiabatic contraction and develops a cuspy profile with the density $\rho \propto r^{-1.5}$. The final distribution of the DM particles in orbits becomes such that there are much more particles that ever come within given radius $r$ than there are within it at any given moment. Whether all DM particles crossing the star surface $\left(r_{\min }<R_{\odot}\right.$ can be finally captured by the star, depends on whether there is enough time for them to lose energy via the DM-nucleon interactions.

\section{Constraints on PBH}

\subsection{Constraints on PBH from star formation}

From now on we would concentrate on particular DM candidate - PBH. Since only the PBHs that can lose energy can be captured, only the trajectories that pass through or in the very near vicinity of the star are relevant for our analysis. To calculate the number of captured PBHs, for each PBH trajectory of this type one has to determine whether there is enough time for the PBH to lose energy and end up in the compact remnant of the star - WD or NS.

There are two stages of the energy loss process. First, the PBH spends part of the time outside the target star at orbits that can be approximately considered as radial, except a small number of cases when the apastron is of order $R_{*}$. The capture time in this case has been estimated in [B] to 
Figure 2: Constraints on the fraction of PBHs as DM. Shaded regions are excluded. The blue shaded regions correspond to the revised constraints derived in this paper assuming the DM densities of $\left(10^{4}, 10^{3}, 10^{2}\right) \mathrm{GeV} / \mathrm{cm}^{3}$ and the velocity dispersion of $7 \mathrm{~km} / \mathrm{s}$ (adopted from [Q] $)$.

be of order

$$
t_{\mathrm{capt}} \simeq 2 \tau \sqrt{\xi_{0}} \sim 2 \times 10^{8} \operatorname{yrs}\left(\frac{10^{22} \mathrm{~g}}{m_{\mathrm{BH}}}\right)
$$

where

$$
\tau=\frac{\pi R_{*}^{5 / 2} v^{2}}{4 G m_{\mathrm{BH}} \sqrt{G M} \ln \Lambda},
$$

with $\xi_{0}=r_{\max } / R_{*}, v$ the escape velocity of the star, $\ln \Lambda$ the Coulomb logarithm that takes a value close to $\ln \Lambda \simeq 30$ for a main-sequence star, and $R_{*}$ and $M$ the radius and the mass of the star, respectively. For the numerical value in eq. (B. l) we have taken $r_{\max } / \bar{R} \simeq 0.1$ corresponding to a value of $\xi_{0} \simeq 4.4 \times 10^{4}$. Thus, the fraction of simulated $\mathrm{PBH}$ orbits that would be captured by the star during its lifetime, could be calculated for each PBH mass.

The second stage starts when $\mathrm{PBH}$ is fully inside the star. It then continues to lose energy through the dynamical friction [ए]] and sinks towards the star center until the moment when the star turns into a compact remnant. If the radius to which the $\mathrm{PBH}$ has been able to sink to during the lifetime of the star is smaller than the radius of the compact remnant, the latter inherits a PBH. The efficiency of the dynamical friction grows with the PBH mass. At large masses all the PBH captured by a star have time to sink to within the radius of the future remnant in a lifetime of the star. At small masses only a fraction of captured $\mathrm{PBH}$ can make it. At the second stage there is no difference with the calculations of Ref. [四]. Combining the two stages gives the fraction of the PBHs that ends up inside the compact remnant.

Finally, the number of simulated trajectories should be related with the mean density of DM in a given environment. This can be done as described in Ref. [四] by calculating the fraction of the DM particles which are gravitationally bound to the prestellar core before the adiabatic contraction. This fraction is proportional to the total DM density and inversely proportional to the cube of the DM velocity dispersion, use of this relation allows to rescale the results for one particular DM density and velocity dispersion to other values of these parameters.

Naturally, most stringent constraints come from observations of compact stars in regions with a high DM density and a low DM velocity dispersion. We consider the values $\rho_{\mathrm{DM}}=$ 
$\left(10^{4}, 10^{3}, 10^{2}\right) \mathrm{GeV} \mathrm{cm}^{-3}$ and $\bar{v}=7 \mathrm{~km} \mathrm{~s}^{-1}$, as a benchmark. The resulting constraints are shown in Fig. $\square$ together with other existing constraints. The strongest constraints shown in light blue correspond to the highest value of DM density considered, i.e $\rho_{\mathrm{DM}} \sim 10^{4} \mathrm{GeV} \mathrm{cm}^{-3}$ and decrease linearly for lower values of DM density. These (rather extreme) conditions could have been present in the cores of metal-poor globular clusters at the epoch of star formation, if they are proved to be of a primordial origin [12] (see detailed discussions in [ [ 9 , []]). Another place where favourable conditions could exist are dwarf spheroidal galaxies that are considered to be DM dominated [13], [4]] and have very low velocity dispersions [[ए3]. However, at present compact objects such as NS or WD have not been observed in dwarf spheroidal galaxiesn although, surveys for pulsars and X-ray binaries have already revealed some hints on NSs existence in dSph galaxies [ए5, [6]].

\section{Conclusions}

The adiabatic contraction of DM during the formation of a star was investigated by simulating the behavior of $\sim 30$ million particles. In particular, the number of particles $n(r)$ within a given radius $r$ was found to be proportional to $r^{1.5}$, which corresponds to the DM density profile $\rho(r) \propto$ $r^{-1.5}$, in agreement with previous calculations and the Liouville theorem.

At the same time, the adiabatic contraction creates a rather special distribution of particle orbits: if one considers the particles that contribute to $n(r)$ for a small $r$, a substantial $(O(1))$ fraction of them have very elongated orbits with periastra smaller than $r$. In fact, the number of particles $v(r)$ that have periastra smaller than $r$ scales as $v(r) \propto r$. Such particles spend only a small fraction of time close to the center, so their individual contributions to the density at small $r$ are suppressed. At $r=R_{\odot}$, there are about $1.8 \times 10^{3}$ more particles that have periastra smaller than $r$ than there are particles within $r$.

This has direct implications for the possible DM capture by stars after their formation. A large number of particles that constitute the DM cusp around the newly-formed star have orbits that cross the star, and that can potentially lead to their capture.

The most stringent constraints are obtained from observations of compact stars in the regions with high DM density and small velocity dispersion. The examples corresponding to the densities $\rho=\left(10^{4}, 10^{3}, 10^{2}\right) \mathrm{GeV} \mathrm{cm}^{-3}$ and a low velocity dispersion $\bar{v}=7 \mathrm{~km} \mathrm{~s}^{-1}$ are shown in Fig. $\square$. Such conditions could have been present at the cores of metal-poor globular clusters at the epoch of star formation if they are of a primordial origin, or - with a DM density somewhat smaller than $10^{3} \mathrm{GeV} / \mathrm{cm}^{3}$ — in dwarf spheroidal galaxies

\section{Acknowledgements}

The work of F.C. and P.T. is supported in part by the IISN and the Belgian Science Policy Belgian Science Policy under IUAP VII/37. The work of M.P. is supported by RSF grant No. 1412-00146. M.P. acknowledges the fellowship of the Dynasty foundation. 


\section{References}

[1] Planck Collaboration, P. A. R. Ade, N. Aghanim, C. Armitage-Caplan, M. Arnaud, M. Ashdown, F. Atrio-Barandela, J. Aumont, C. Baccigalupi, A. J. Banday, and et al., Planck 2013 results. XVI. Cosmological parameters, ArXiv e-prints (Mar., 2013) [arXiv: 1303.5076].

[2] C. Kouvaris and P. Tinyakov, Growth of Black Holes in the interior of Rotating Neutron Stars, arxiv: 1312.3764 .

[3] F. Capela, M. Pshirkov, and P. Tinyakov, Constraints on primordial black holes as dark matter candidates from capture by neutron stars, Phys.Rev. D87 (2013) 123524, [arXiv:1301.4984].

[4] F. Capela, M. Pshirkov, and P. Tinyakov, Constraints on Primordial Black Holes as Dark Matter Candidates from Star Formation, Phys.Rev. D87 (2013) 023507, [GrXiv:1209.6021].

[5] G. Jungman and M. Kamionkowski, Neutrinos from particle decay in the Sun and Earth, Phys. Rev. D 51 (Jan., 1995) 328-340, [hep-ph/9407351].

[6] S. Nussinov, L.-T. Wang, and I. Yavin, Capture of inelastic Dark Matter in the sun, JCAP 8 (Aug., 2009) 37, [arXiv:0905.1333].

[7] C. Kouvaris and P. Tinyakov, Constraining Asymmetric Dark Matter through observations of compact stars, Phys.Rev. D83 (2011) 083512, [arXiv:1012.2039].

[8] S. Tremaine and J. Gunn, Dynamical Role of Light Neutral Leptons in Cosmology, Phys.Rev.Lett. 42 (1979) 407-410.

[9] F. Capela, M. Pshirkov, and P. Tinyakov, Adiabatic contraction revisited: Implications for primordial black holes, Phys. Rev. D 90 (Oct., 2014) 083507, [arXiv: 1403.7098].

[10] P. Ullio, H. Zhao, and M. Kamionkowski, A Dark matter spike at the galactic center?, Phys.Rev. D64 (2001) 043504, [astro-ph/0101481].

[11] S. Chandrasekhar, Brownian Motion, Dynamical Friction, and Stellar Dynamics, Reviews of Modern Physics 21 (July, 1949) 383-388.

[12] G. Bertone and M. Fairbairn, Compact Stars as Dark Matter Probes, Phys.Rev. D77 (2008) 043515, [arxiv:0709.1485].

[13] L. E. Strigari, S. M. Koushiappas, J. S. Bullock, M. Kaplinghat, J. D. Simon, M. Geha, and B. Willman, The Most Dark-Matter-dominated Galaxies: Predicted Gamma-Ray Signals from the Faintest Milky Way Dwarfs, ApJ 678 (May, 2008) 614-620, [GrXiv:0709.1510].

[14] L. E. Strigari, S. M. Koushiappas, J. S. Bullock, and M. Kaplinghat, Precise constraints on the dark matter content of MilkyWay dwarf galaxies for gamma-ray experiments, Phys. Rev. D 75 (Apr., 2007) 083526, [astro-ph/0611925].

[15] E. Rubio-Herrera and T. Maccarone, Pulsar searches in nearby dwarf spheroidal galaxies, in IAU Symposium, vol. 291 of IAU Symposium, pp. 111-114, Mar., 2013.

[16] T. J. Maccarone, A. Kundu, S. E. Zepf, A. L. Piro, and L. Bildsten, The discovery of X-ray binaries in the Sculptor dwarf spheroidal galaxy, MNRAS 364 (Nov., 2005) L61-L65, [astro-ph/. 